Ambiente \& Água - An Interdisciplinary Journal of Applied Science
ISSN 1980-993X - doi:10.4136/1980-993X
www.ambi-agua.net
E-mail: ambi-agua@agro.unitau.br

\title{
Estudo energético e ambiental da substituição de moinho de polpa de celulose como ganho ambiental
}

\author{
doi: 10.4136/ambi-agua.1366
}

Received: 16 Aug. 2013; Accepted: 29 Nov. 2013

\author{
${\text { Francisco Savastano } \text { Neto }^{1 *} \text {; Nelson Wellausen Dias }}^{1,2}$ \\ ${ }^{1}$ Programa de Pós-graduação em Ciências Ambientais (PPG-CA) \\ Universidade de Taubaté, Taubaté, SP, Brasil \\ ${ }^{2}$ Instituto Brasileiro de Geografia e Estatística (IBGE), Aracaju, SE, Brasil \\ *Autor correspondente: e-mail: fsavastano@uol.com.br, \\ nwdias@gmail.com
}

\section{RESUMO}

Uma porção significativa da energia gasta no Brasil está associada ao consumo industrial (40,6\%). O objetivo desta pesquisa é demonstrar uma dentre as diversas formas de reduzir o consumo de energia elétrica pela indústria e, consequentemente, reduzir as emissões associadas com a geração, transmissão e consumo desse recurso. A busca pela eficiência energética, aliada com práticas econômicas, sociais e ambientais, pode mudar o rumo da indústria na direção à sustentabilidade. As emissões de $\mathrm{CO}_{2} / \mathrm{kWh}$ foram escolhidas como indicador de sustentabilidade para monitorar o padrão de consumo de energia durante o desenvolvimento desta pesquisa. O objetivo principal foi inferir a redução na emissão de gases de efeito estufa associada com uma estratégia de incremento da eficiência energética adotada por uma indústria de grande porte. Nessa indústria, um sistema de moinho de polpa de celulose, usado em um processo de fabricação de fraldas descartáveis, foi substituído por outro sistema de maior eficiência. A redução do consumo de energia alcançou $60 \%$, e a redução na emissão de gases de efeito estufa foi estimada em 80 ton/ano de dióxido de carbono $\left(\mathrm{CO}_{2}\right)$. Esses resultados demonstram a relação entre a adoção de sistemas mais eficientes de energia com a redução na emissão de gases de efeito estufa, o que, consequentemente, contribui para a redução dos custos na indústria.

Palavras-chave: eficiência energética, indicadores de sustentabilidade, redução de emissões de gases do efeito estufa.

\section{Energy and environmental study of the replacement of a cellulose pulp mill as an environmental gain}

\begin{abstract}
A significant fraction of the energy spent in Brazil is associated with industrial consumption (40.6\%). The purpose of this work was to demonstrate one of many ways to reduce electric energy consumption by the industry and, consequently, reduce emissions associated with the generation, transmission, and consumption of this resource. The search for energetic efficiency, coupled with economic, social, and environmental practices can shift the industry in the direction of sustainability. The emission of $\mathrm{CO}_{2} / \mathrm{kWh}$ was chosen as a sustainability indicator to monitor the energy consumption pattern during the development of
\end{abstract}


this research. The main objective was to assess the reduction of greenhouse gas emissions associated with a more efficient electric energy consumption strategy adopted by a large manufacturer. At this company, a cellulose pulp milling system used during a diaper manufacturing process was replaced by another system with higher efficiency. Energy consumption was reduced by $60 \%$, and greenhouse gas emissions were reduced by an estimated 80 ton/year of carbon dioxide $\left(\mathrm{CO}_{2}\right)$. These results demonstrate the relationship between adopting a more energy efficient system and a reduction in greenhouse gas emissions. Moreover, these practices contribute to significant industrial cost reduction.

Keywords: energy efficiency, sustainability indicator, greenhouse gas emissions reduction.

\section{INTRODUÇÃO}

Após a Revolução Industrial, iniciada na Inglaterra em meados do século XVIII, passouse a utilizar a energia dos combustíveis fósseis. A partir daquela época, seu uso vem se intensificando, e atualmente a energia é um insumo básico em toda a cadeia produtiva.

Processos produtivos desenvolvidos em uma época em que a energia era abundante e barata precisam ser revistos, pois, além da escassez e do alto custo, o uso indiscriminado dessa energia está aumentando de forma descontrolada a emissão de gases de efeito estufa (GEE). Nos últimos anos, a quantificação dessas emissões vem se tornando um importante indicador de sustentabilidade na indústria, por exemplo, na indústria de petróleo e gás (Amaral, 2002). A prática de usar indicadores de sustentabilidade nas indústrias traz benefícios ligados à transparência, à imagem da empresa no mercado e ao monitoramento do desempenho econômico, social e ambiental ao longo do tempo (Amaral, 2004).

A forma organizacional da sociedade faz com que essa energia venha de pontos cada vez mais distantes da sua extração ou geração, tornando complexa a cadeia de fornecimento, aumentando as perdas nos processos de transformação e transporte, além de impactar o ambiente. $\mathrm{O}$ uso eficiente da energia reduz custo, adia investimentos em infraestrutura de fornecimento de energia e funciona como um componente de conservação do meio ambiente.

Hoje, o consumo de combustível está intimamente relacionado ao nível de desenvolvimento de uma nação ou região. Segundo Goldemberg e Lucon (2007), na maioria dos países onde o consumo de energia comercial per capita está abaixo de uma tonelada equivalente de petróleo (tep) por ano, as taxas de analfabetismo, mortalidade infantil e fertilidade total são altas, enquanto a expectativa de vida é baixa. Ultrapassar a barreira de uma tep / per capita parece ser, portanto, essencial para o desenvolvimento. À medida que o consumo de energia comercial per capita aumenta para valores de duas tep, ou mais, como é o caso dos países desenvolvidos, as condições sociais melhoram consideravelmente. Já os países em desenvolvimento não podem pensar em atingir esse patamar, sem respeitar as limitações impostas pelo meio ambiente, a exemplo do que ocorreu com os países desenvolvidos, uma vez que os danos ambientais seriam imensos.

De acordo com os critérios mencionados, o Brasil é considerado um país desenvolvido, nesse setor, pois a média de consumo de energia per capita anual é de 2,5 tep, entretanto a distribuição do consumo dessa energia no país é feita de forma desigual. No Brasil, o setor industrial é o maior consumidor de recursos naturais. O Quadro 1 mostra o consumo final de energia, segmentado por setores econômicos, além da evolução do consumo de energia nos anos de 2006 e 2007. Conforme os dados do BEN - Balanço Energético Nacional (Brasil, 2008), em 2007 o consumo de energia pelo setor industrial representou 40,6\% do total da energia consumida no país.

Reduzir o consumo de energia do setor industrial e promover o uso eficiente da energia são contribuições para diminuir a pressão na necessidade de oferta de energia primária. Os benefícios podem ser significativos, tanto do ponto de vista ambiental, pela redução nas 
emissões de GEE, como também do ponto de vista econômico, devido ao menor desembolso a ser pago pela energia consumida.

Neste trabalho, objetivou-se avaliar o consumo de energia em dois moinhos de polpa de celulose: um moinho antigo, fabricado segundo os conceitos vigentes na época de oferta de energia barata, e outro moinho de nova geração, mais eficiente do ponto de vista energético, além de quantificar a redução nas emissões de $\mathrm{CO}_{2}$.

Quadro 1. Consumo de energia por setores da economia brasileira.

\begin{tabular}{|cccc|}
\hline \multicolumn{3}{c|}{$10^{3}$ tep } \\
\hline SETOR & $\mathbf{2 0 0 7}$ & $\mathbf{2 0 0 6}$ & VARIAÇÃO \% \\
\hline INDUSTRIAL & 81752 & 76757 & 6,5 \\
TRANSPORTES & 56894 & 53270 & 6,8 \\
RESIDENCIAL & 22601 & 22090 & 2,3 \\
ENERGÉTICO ${ }^{1}$ & 21478 & 18823 & 14,1 \\
AGROPECUÁRIO & 9104 & 8550 & 6,5 \\
COMERCIAL & 5893 & 5545 & 6,3 \\
PÚBLICO & 3494 & 3453 & 1,2 \\
\hline TOTAL & $\mathbf{2 0 1 2 1 6}$ & $\mathbf{1 8 8 5 7 4}$ & $\mathbf{6 , 7}$ \\
\hline
\end{tabular}

Fonte: Balanço Energético Nacional (MME, 2008).

${ }^{1}$ Agrega os centros de transformação e/ou processos de extração e transporte interno de produtos energéticos na sua forma final.

Eficiência energética é a capacidade de gerar, distribuir e consumir a energia da forma mais racional possível. Quando se pensa em eficiência energética, deve-se ter em mente a constante evolução, a contínua busca por fazer cada vez mais com menos energia. Esse conceito pode e deve ser estendido a todo e qualquer recurso natural, pois sempre será possível melhorar a eficiência de um processo ou sistema.

Ocorre que, para suprir essa demanda sempre crescente por energia, os sistemas tornaram-se complexos, exigindo variados e repetidos processos de transformações. A cada etapa de transformação que um energético é submetido, uma parcela de perda é produzida. As perdas vão se somando, até o consumo final.

Cabe à indústria, como usuária final, trabalhar em seus processos para garantir o uso eficiente de qualquer energético. Caldeiras, sistemas de ar comprimido ou transporte pneumático, sistema de refrigeração ou acionamentos elétricos por motores devem ser constantemente avaliados, para garantir as operações com máxima eficiência energética.

Jannuzzi (2002) identifica os benefícios que a sociedade pode obter com a implementação de técnicas que conduzam ao caminho da eficiência energética:

- Reduzir ou adiar as necessidades de investimento em geração, transmissão e distribuição;

- Reduzir impactos ambientais locais e globais, principalmente os relacionados com a produção de eletricidade;

- Reduzir os custos da energia para o consumidor final;

- Contribuir para aumentar a confiabilidade do sistema elétrico.

Em seu trabalho, Esparta (2008) avalia o contexto atual das ações para mitigar as mudanças adversas do clima e a fase de transição do setor elétrico brasileiro, identificando como reduzir as emissões da linha de base para a geração de eletricidade a partir de fontes 
renováveis, e adoção do Mecanismo de Desenvolvimento Limpo como ferramenta de planejamento para o setor elétrico.

Nesta pesquisa, buscou-se substituir o moinho por outro equipamento mais eficiente, para diminuir a demanda por energia elétrica $(\mathrm{kWh})$ que coloca esse conjunto em operação. Com isso, pretendeu-se reduzir a emissão de gases de efeito estufa $\left(\mathrm{tCO}_{2}\right)$ associada à geração, transporte e consumo de energia elétrica, melhorando assim a eficiência energética $(\mathrm{kW} / \mathrm{kg})$ do equipamento.

\section{MATERIAL E MÉTODO}

Para a formulação de uma estratégia de gerenciamento do uso da energia como forma de redução dos impactos ambientais, o trabalho de eficientização energética foi desenvolvido em uma empresa fabricante de produtos descartáveis para higiene e saúde. Essa empresa, que possui várias linhas de produção de fraldas descartáveis, localiza-se no eixo Rio-São Paulo.

Uma máquina que produz fraldas descartáveis é composta por vários sistemas, e alguns deles são acionados por motores de grande porte, que consomem muita energia. $\mathrm{O}$ moinho em que é feito o desfibramento da polpa de celulose e que tem seu conjunto acionado por um motor de $250 \mathrm{CV}$ foi o sistema estudado.

A Figura 1 mostra o esquema do sistema de formação de uma fralda descartável. A polpa triturada no moinho é conduzida, para a roda formadora, pelo vácuo produzido pelo ventilador, e um sistema de válvula dumper ajusta o nível do vácuo na roda de formação. $\mathrm{O}$ ar que transporta a polpa passa pelo ventilador e, como está contaminado com polpa antes de ser lançado na atmosfera, passa por um sistema de filtragem. O painel formado na roda é destacado e conduzido para completar o processo de fabricação da fralda descartável.

ESQUEMA DO SISTEMA DE FORMAÇÃO DA FRALDA

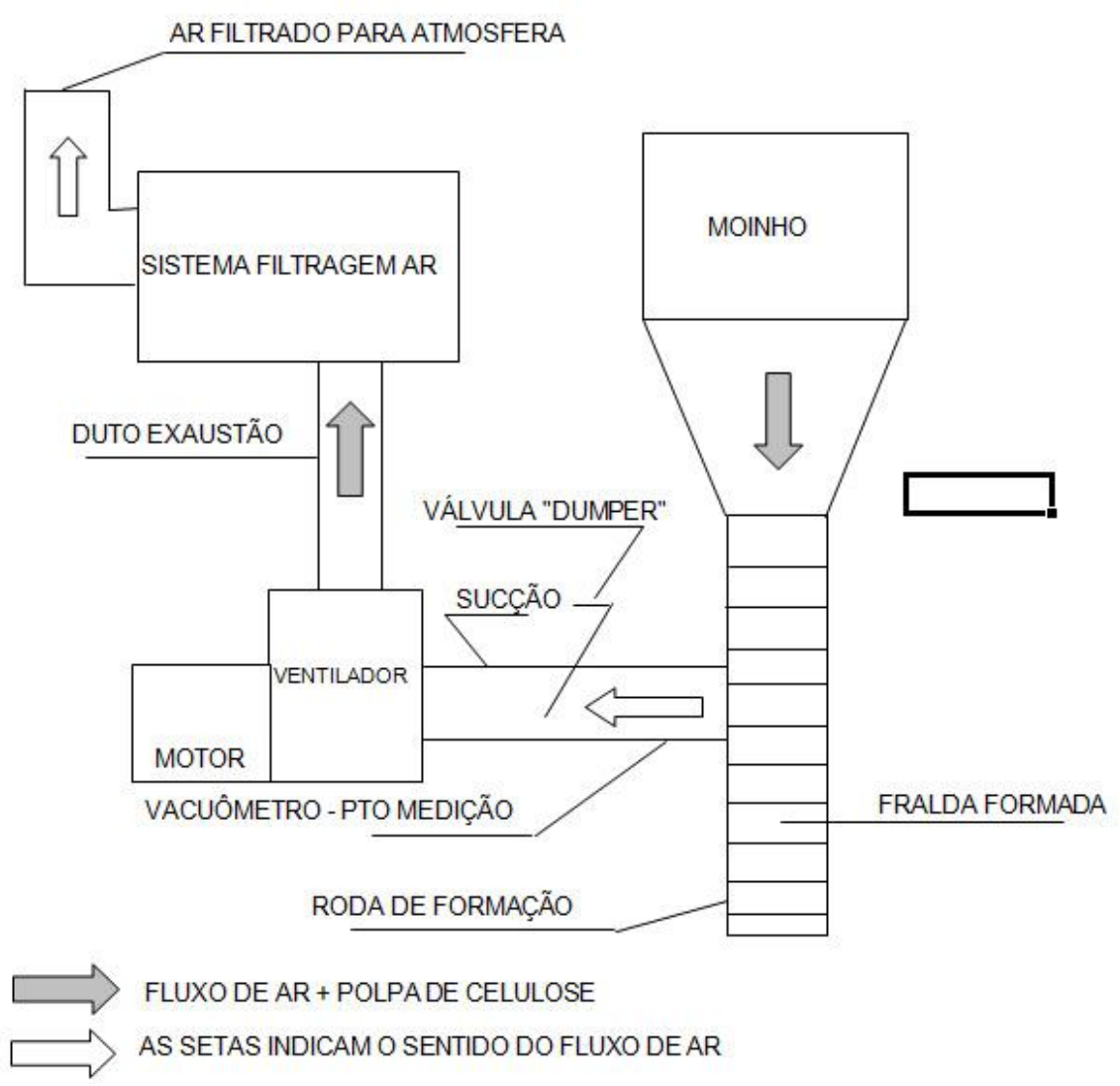

Figura 1. Esquema do sistema de formação da fralda. 
Na fralda descartável, a polpa de celulose, um dos insumos importantes usado no processo produtivo, para ser incorporada ao produto deve ser triturada ou desfibrada em moinhos. Originalmente, essa operação de desfibramento era realizada em moinhos que estavam em operação há mais de 20 anos e que apresentavam baixo rendimento operacional. Um desses moinhos antigos, de martelo oscilante, foi substituído por um sistema mais eficiente, que incorpora conceitos atuais de desfibramento, aliado a um sistema de acionamento elétrico de alto rendimento (apenas 60CV). Desse modo, o novo conjunto necessita menor quantidade de energia para realizar o mesmo trabalho, ou seja, abastecer a linha de produção de fraldas com a quantidade necessária de celulose desfibrada.

Os dados coletados para avaliação dos sistemas foram: velocidade de produção (em produtos por minuto), massa do produto (gramas), tensão de alimentação do moinho (volts), corrente consumida pelos moinhos (ampères); temperatura da carcaça do moinho (graus centígrados), temperatura ambiente (graus centígrados) e data e responsável pela medição.

As medidas foram simultâneas em uma unidade com o moinho antigo e em outra com o moinho de nova geração. Com os dados coletados foram elaborados os gráficos comparativos para os dois moinhos, mostrando o consumo de energia, a quantidade de polpa desfibrada e a eficência energética.

As emissões de carbono associadas à geração de energia variam conforme a base energética de cada país. Segundo a Agência Internacional de Energia (IEA, 2012), os combustíveis mais usados no Brasil para geração de energia emitem os seguintes valores de $\mathrm{CO}_{2} / \mathrm{kWh}$ : carvão - $920 \mathrm{~g}$; gás natural $-400 \mathrm{~g}$; e, óleo combustível $-670 \mathrm{~g}$. Com base nesses valores, o Brasil emitiu, em 2010: 51,9 milhões de toneladas de $\mathrm{CO}_{2}$, pelo uso do carvão na geração de energia; 51,8 milhões de toneladas de $\mathrm{CO}_{2}$, pelo uso do gás natural; e, 284 milhões de toneladas de $\mathrm{CO}_{2}$, pelo uso do óleo combustível (IEA, 2012). Segundo Koch (2002), as emissões de $\mathrm{CO}_{2} / \mathrm{kWh}$ no setor hidroelétrico variam de 2 a $48 \mathrm{~g}$ de gases de efeito estufa.

Para o cálculo das emissões de $\mathrm{CO}_{2}$ relativas à produção e consumo de energia elétrica para os moinhos foram adotadas as seguintes premissas: as linhas de produção para produtos descartáveis trabalham por 6610 horas no período de um ano, e as emissões médias de $\mathrm{CO}_{2}$ adotadas para o setor elétrico são de $283 \mathrm{~g}$ de $\mathrm{CO}_{2} / \mathrm{kWh}$, para as regiões Sul/Sudeste/CentroOeste (Esparta, 2008).

\section{RESULTADOS E DISCUSSÃO}

As Figuras 2 e 3 mostram os gráficos com o consumo de energia $(\mathrm{kW})$, consumo de polpa $(\mathrm{kg} / \mathrm{h})$ e eficiência energética $(\mathrm{kg} / \mathrm{kW})$ para o moinho antigo e para o moinho de nova geração, respectivamente. Observa-se menor necessidade de energia e menor potência de acionamento para o moinho novo.

Os valores mostrados no Quadro 2 indicam que a eficiência energética do moinho novo é $147 \%$ superior à do moinho antigo, ou seja, desfibra uma quantidade maior de polpa para a mesma quantidade de energia.

A partir dos dados considerados para se estimar a emissão de gases de efeito estufa, elaborou-se um comparativo de emissões para os dois moinhos (Quadro 3). O início de operação da nova geração do moinho de polpa possibilitou a redução na emissão de $\mathrm{CO}_{2}$ de 80 t/ano, em função da redução do consumo da energia elétrica (60\%). 


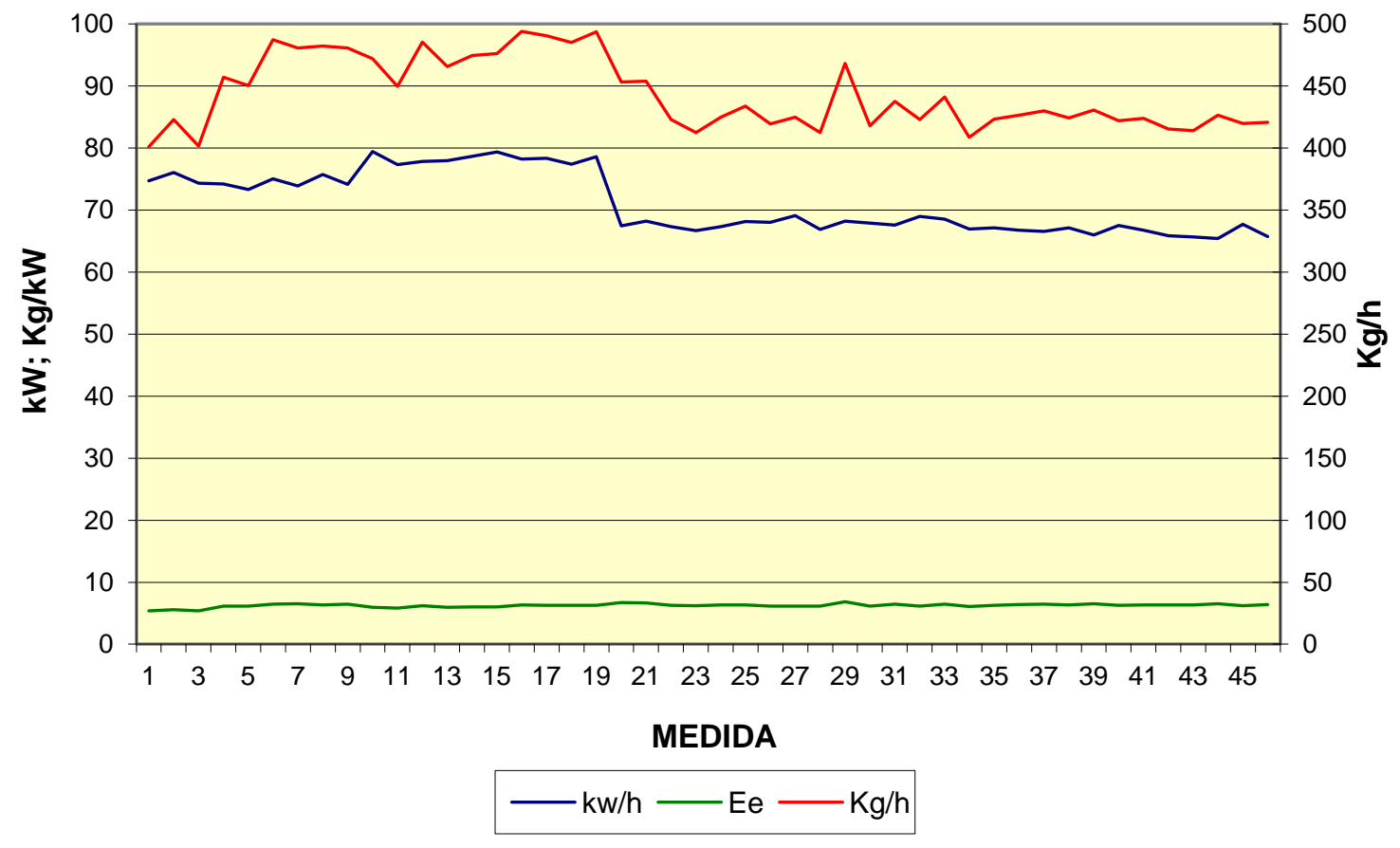

Figura 2. Gráfico: eficiência energética do moinho antigo.

Nota: os valores de potência $(\mathrm{kW})$ e eficiência energética $(\mathrm{kg} / \mathrm{kW})$ variam na escala de $0-100$.

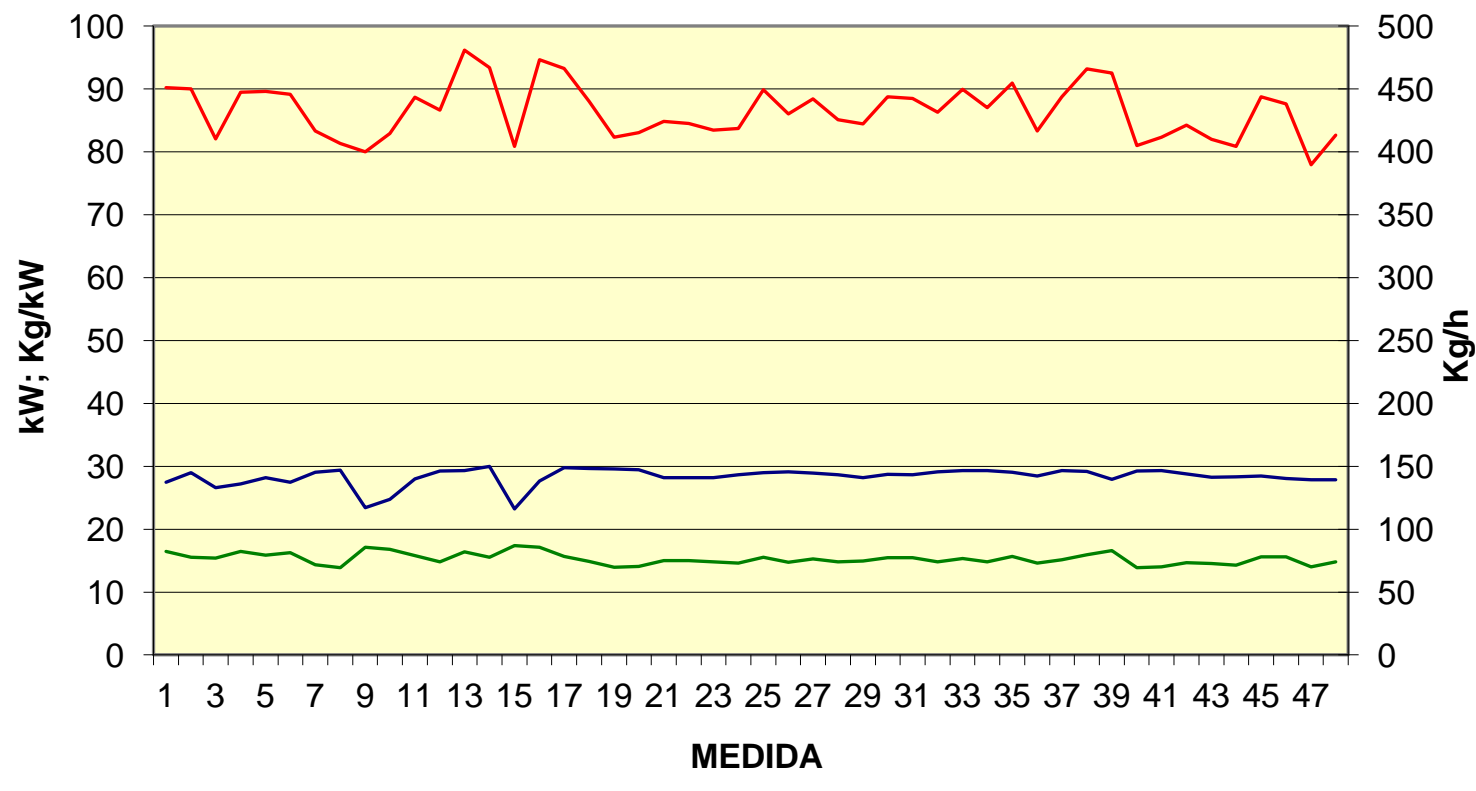

$$
-\mathrm{Kw} / \mathrm{h}-\mathrm{Kg} / \mathrm{kW}-\mathrm{Kg} / \mathrm{h}
$$

Figura 3. Gráfico: eficiência energética do moinho da nova geração.

Nota: os valores de potência $(\mathrm{kW})$ e eficiência energética $(\mathrm{kg} / \mathrm{kW})$ variam na escala de $0-100$. 
Quadro 2. Comparativo da eficiência energética dos moinhos em operação.

\begin{tabular}{|lccc|}
\hline \multicolumn{4}{|c|}{ COMPARATIVO DE EFICIÊNCIA ENERGÉTICA ENTRE OS DOIS MOINHOS } \\
\hline & Ee Kg / kW & $\begin{array}{c}\text { DESVIO } \\
\text { PADRÃO }\end{array}$ & CONSUMO kWh \\
\hline MOINHO POLPA ANTIGO & 6,2 & 0,29 & 71,1 \\
NOVA GERAÇÃO MOINHO POLPA & 15,3 & 0,89 & 28,3 \\
VARIAÇÃO \% & 147 & - & 60 \\
\hline
\end{tabular}

Além da redução das emissões de $\mathrm{CO}_{2}$, foram obtidos outros benefícios, como a redução no nível de ruído, redução no custo de produção gerado pela diminuição no uso de um importante insumo do processo produtivo (a energia elétrica), diminuição das manutenções nos moinhos, e o aumento na qualidade final do produto em função de melhor qualidade da polpa desfibrada.

Quadro 3. Emissões anuais de $\mathrm{CO}_{2}$ por moinho, em uma linha de produção.

\begin{tabular}{|lccc|}
\hline \multicolumn{4}{|c|}{ EMISSÕES DE CO2 (POR LINHA DE PRODUÇÃO) } \\
\hline & $\begin{array}{c}\text { CONSUMO } \\
\text { kWh }\end{array}$ & $\begin{array}{c}\text { CONSUMO ANUAL } \\
\text { kWh/ano }\end{array}$ & $\begin{array}{c}\text { EMISSÃO CO2 } \\
\text { tCO2/ano }\end{array}$ \\
\hline MOINHO POLPA ANTIGO & 71,1 & 469971 & 133,0 \\
NOVA GERAÇÃO MOINHO POLPA & 28,3 & 187063 & 52,9 \\
REDUÇÃO CO2 / ANO & - & - & 80 \\
\hline
\end{tabular}

\section{CONCLUSÃO}

Pode-se observar, pelos dados apresentados, que os objetivos de redução no consumo de energia elétrica, com a consequente redução nas emissões de $\mathrm{CO}_{2}$, foram atingidos. Esse tipo de ação gera ganho econômico, pois com a economia de energia é obtida uma redução no custo do processo produtivo, e há ganho ambiental, uma vez que reduz as emissões de gases de efeito estufa. Esse fator nem sempre é considerado na hora de planejar investimentos e ações empresariais. Neste estudo podem-se evidenciar os ganhos econômicos e ambientais a partir do monitoramento simultâneo de equipamentos distintos. Somente ações desse tipo viabilizam quantificar esses ganhos de forma objetiva e transparente.

Como visão de futuro, é oportuna a colocação de mais alguns pontos que possam ajudar no questionamento sobre o uso adequado e eficiente da energia, em um país com grande diversidade cultural e recursos naturais. As estratégias energéticas deverão ser definidas, pelos governos e empresas do setor privado, buscando sempre uma matriz energética com baixas emissões de gases de efeito estufa, que de fato usem todas as formas de energia disponíveis localmente. Isso porque a diversificação energética pode ser $\mathrm{o}$ caminho para $\mathrm{o}$ desenvolvimento sustentável.

Com o uso eficiente da energia é possível reduzir e até mesmo postergar as necessidades de investimentos em geração, transmissão e distribuição, com a consequente redução do impacto ambiental ocasionado por toda a cadeia de fornecimento de energia.

\section{REFERÊNCIAS}

AMARAL, S. P. Indicadores de sustentabilidade ambiental, social e econômica: uma proposta para a indústria de petróleo brasileira. Meio Ambiente Industrial, v. 39, p. 38, 2002. 
AMARAL, S. P. Sustentabilidade ambiental, social e econômica nas empresas: como entender, medir e relatar. São Paulo: Tocalino, 2004. 126 p.

BRASIL. Ministério de Minas e Energia. Balanço energético nacional. Brasília, 2008. Disponível em: < http://ben.epe.gov.br/>. Acesso em: jan. 2009.

ESPARTA, A. R. J. Redução de gases do efeito estufa no setor elétrico brasileiro: a experiência do mecanismo de desenvolvimento limpo do Protocolo de Quioto, e uma visão futura. 2008. Disponível em:

<http://de.scientificcommons.org/adelino_ricardo_jacintho_esparta>. Acesso em maio 2009.

GOLDEMBERG, J.; LUCON, O. Energia e o meio ambiente no Brasil. São Paulo: USP, 2007. p. 7-20. (Estudos Avançados, 59)

INTERNATIONAL ENERGY AGENCY - IEA. $\mathbf{C O}_{2}$ emissions from fuel combustion highlights. Disponível em: http://www.iea.org/co2highlights/co2highlights.pdf. Acesso em: nov. 2012 .

JANNUZZI, G. M. Aumentando a eficiência nos usos finais de energia no Brasil. 2002. Disponível em: <http://www.fem.unicamp.br/ jannuzzi/documents/unicamp-20anos.pdf>. Acesso em jul. 2009.

KOCH, F. H. Hydropower - internalised costs and externalised benefits. In: WORKSHOP ON EXTERNALITIES AND ENERGY POLICY: THE LIFE CYCLE ANALYSIS APPROACH, 15-16 de novembro de 2001, Paris. Anais... Paris: Organisation for Economic Co-Operation and Development, 2002. p. 131-140. 